The Astrophysical Journal, 579:L17-L20, 2002 November 1

(C) 2002. The American Astronomical Society. All rights reserved. Printed in U.S.A.

\title{
CARBON ABUNDANCES OF FAINT STARS IN M13: EVIDENCE OF TWO ABUNDANCE-ALTERING MECHANISMS ${ }^{1}$
}

\author{
Michael M. BRILEY \\ Department of Physics and Astronomy, University of Wisconsin Oshkosh, 800 Algoma Boulevard, Oshkosh, WI 54901; mike@maxwell.phys.uwosh.edu \\ JUdith G. COHEN \\ Palomar Observatory, California Institute of Technology, MS 105-24, Pasadena, CA 91125 \\ AND \\ Peter B. Stetson ${ }^{2}$ \\ Dominion Astrophysical Observatory, Herzberg Institute of Astrophysics, National Research Council, 5071 West Saanich Road, \\ Victoria, BC V9E 2E7, Canada; peter.stetson@nrc.ca \\ Received 2002 August 20; accepted 2002 September 26; published 2002 October 10
}

\begin{abstract}
We present an analysis of $\mathrm{CH}$ band strengths in Keck Low-Resolution Imaging Spectrometer spectra of a sample of 81 stars in M13 within 2 mag of the main-sequence turnoff. The subgiants clearly exhibit a substantial (a factor of 6) spread in [C/Fe]. Moreover, the bulk of the subgiants possess $\mathrm{C}$ abundances larger than those found among their more luminous counterparts. The turnoff stars themselves are too warm for appreciable $\mathrm{CH}$ formation, but the relatively small range in the observed $\mathrm{CH}$ band strength for stars just below the turnoff nevertheless translates into this same spread in $[\mathrm{C} / \mathrm{Fe}]$. Still fainter, the sample size is small, but the same range in $[\mathrm{C} / \mathrm{Fe}]$ appears to be present. On the basis of these observations we suggest that a process external to the present stars has resulted in a substantial star-to-star dispersion in $[\mathrm{C} / \mathrm{Fe}]$ (and possibly other light elements) among all stars in M13. In addition, the surface $\mathrm{C}$ abundances among the more luminous stars have been further modified by the operation of an internal deep-mixing mechanism during red giant branch ascent. The amplitude of the scatter we find in $[\mathrm{C} / \mathrm{Fe}]$ at all luminosities may prove difficult to explain via accretion from intermediatemass asymptotic giant branch stars as the external "polluting" mechanism.
\end{abstract}

Subject headings: globular clusters: general — globular clusters: individual (M13) — stars: abundances — stars: evolution

\section{INTRODUCTION}

A detailed history of the 30 years of progress in our understanding and interpretation of the compositions of globular cluster stars is far too long and complex to offer in the present work. Hence, we present below only the shortest of summaries. For a more proper treatment, we recommend to the reader the more comprehensive reviews of Kraft (1994) and Da Costa (1998).

Contrary to the canonical picture of globular clusters as chemically homogeneous populations of stars, significant starto-star differences in the abundances of $\mathrm{C}, \mathrm{N}$, and often other light elements associated with proton capture reactions have been found in every Galactic globular cluster studied to date. Locked within these inhomogeneities appears to be at least a partial history of processes at work in the early cluster environment. However, these patterns are also likely subject to further modification driven by a less than well-understood mixing mechanism at work within the stars themselves. With this in mind, intracluster abundance inhomogeneities are usually discussed in terms of these two distinct origins: internal post-first-dredge-up "deep mixing" or a process external to the present-day cluster stars (e.g., the accretion of ejecta from more massive stars).

In the case of deep mixing, material exposed to the $\mathrm{CN}$ (and possibly $\mathrm{ON}$ ) cycle regions above the H-burning shell of an

\footnotetext{
${ }^{1}$ Based on observations obtained at the W. M. Keck Observatory, which is operated jointly by the California Institute of Technology, the University of California, and the National Aeronautics and Space Administration.

${ }^{2}$ Guest investigator of the UK Astronomy Data Centre; and Visiting Astronomer, Canada-France-Hawaii Telescope, operated by the National Research Council of Canada, the Centre National de la Recherche Scientifique de France, and the University of Hawaii.
}

evolving low-mass star is circulated into the outer convective envelope during red giant branch (RGB) ascent resulting in increased surface $\mathrm{N}$ abundances at the expense of $\mathrm{C}$ (and possibly O). Observational evidence that such a process operates can be found among low-mass metal-poor $(-2 \leq[\mathrm{Fe} / \mathrm{H}] \leq-1)$ field giants, where Gratton et al. (2000) note lowered $\mathrm{C}$ abundances, enhanced $\mathrm{N}$, depleted $\mathrm{Li}$, and lower ${ }^{12} \mathrm{C} /{ }^{13} \mathrm{C}$ ratios than expected from first dredge-up (see also Charbonnel \& do Nascimento 1998; Keller, Pilachowski, \& Sneden 2001). Mixing may be even more efficient in the metal-poor $(-2 \approx[\mathrm{Fe} / \mathrm{H}])$ globulars, where substantial decreases in $[\mathrm{C} / \mathrm{Fe}](\approx 1 \mathrm{dex})$ with increasing luminosity have been observed along the RGBs of M92 (Bellman et al. 2001), M15 (Trefzger et al. 1983), and NGC 6397 (Briley et al. 1990). Mixing has also been suggested as an explanation for the detections of $\mathrm{O}$ underabundances and $\mathrm{O}-\mathrm{Na}, \mathrm{Al}-\mathrm{Mg}$ anticorrelations among the bright giants of numerous globular clusters, e.g., M5, M3, M10, M13 (see the composite figure for O-Na relations assembled by Ramírez \& Cohen [2002a and references therein], their Fig. 12) - trends notably absent from the field population (Gratton et al. 2000). If proton capture reactions on $\mathrm{Ne}$ and $\mathrm{Mg}$ resulting in $\mathrm{Na}$ and $\mathrm{Al}$ are also operating at $\mathrm{ON}$ cycle temperatures (Cavallo \& Nagar 2000 and references therein), one could then expect the abundance patterns observed in most cluster giants to be at least qualitatively reproduced under the conditions of sufficiently deep mixing.

But the underlying physical process connecting the shell region with the envelope beyond first dredge-up is not included in standard models of low-mass stellar evolution and remains poorly understood. Some possible mechanisms explored include meridional circulation (Sweigart \& Mengel 1979), various treatments of diffusion (e.g., Charbonnel 1995; Denissenkov \& Tout 2000), and shell flashes (Aikawa, Fujimoto, \& Kato 2001)—see 
the review of the theory of RGB stars by Salaris, Cassisi, \& Weiss (2002) for a more thorough discussion.

As early as 24 years ago it was known that the subgiant branch (SGB) stars and likely the main-sequence (MS) stars of the metal-rich $([\mathrm{Fe} / \mathrm{H}]=-0.8)$ cluster 47 Tuc exhibit $\mathrm{C}$ and $\mathrm{N}$ differences similar to those found on its RGB (Hesser 1978). These results have been confirmed by Cannon et al. (1998 and references therein), and significant C/N inhomogeneities among SGB and MS stars have since been observed in NGC 6752, M5 (Suntzeff \& Smith 1991; Cohen, Briley, \& Stetson 2002), and M71 (Cohen 1999b). Variations in other light elements have also been noted among the SGB/MS stars of these very clusters: Na-CN correlated on the MS of 47 Tuc (Briley et al. 1996), O-Na and $\mathrm{Mg}-\mathrm{Al}$ anticorrelated among the MS stars of NGC 6752 (Gratton et al. 2001), and O-Na anticorrelated in M71 SGB stars (Ramírez \& Cohen 2002a). Most recently, Ramírez \& Cohen (2002b) have shown a significant dispersion in Na extending from the M5 RGB to its MS turnoff (MSTO). These inhomogeneities are of course difficult to explain within the framework of mixing theories and imply that some component of the abundance differences is in place well before appreciable RGB ascent, possibly embedded in the present-day stars much earlier in the cluster history. Indeed, the nuclei observed to vary among the SGB/MS stars, i.e., C, $\mathrm{N}, \mathrm{O}, \mathrm{Na}, \mathrm{Al}$, and $\mathrm{Mg}$, suggest as a source the hot bottomburning regions of intermediate-mass asymptotic giant branch (AGB) stars (Ventura et al. 2001). As put forth by Cottrell \& Da Costa (1981), the proton-exposed ejecta from such stars may then have been accreted by and thus "polluted" the presentday cluster stars (see Cannon et al. 1998 for a more thorough discussion).

It has become increasingly clear that a full description of cluster abundance inhomogeneities requires an understanding of the extent of the contributions of both deep internal mixing and external sources of chemical differences. Unfortunately, the same reaction chains (proton captures on $\mathrm{C}, \mathrm{N}, \mathrm{O}, \mathrm{Ne}$, and $\mathrm{Mg}$ ) appear to be associated with both mechanisms. Perhaps the only method of disentangling the role of each process is to compare the abundances of the less luminous cluster stars with their more evolved counterparts. Because of the strength of the $\mathrm{CH}$ band, for technical reasons this is easier to accomplish at present for $\mathrm{C}$ than for the other light elements listed above.

In this regard, the globular cluster M13 provides us with a particularly interesting target. With an overall metallicity of $[\mathrm{Fe} / \mathrm{H}]=-1.51 \mathrm{dex}$ (Kraft et al. 1992), it is perhaps one of the most thoroughly studied "intermediate-metallicity" clusters. It has long been known to contain giants with a large and bimodal spread in $\mathrm{CN}$ band strengths, anticorrelated with $\mathrm{CH}$, whose origin lies in an apparent C-N anticorrelation (e.g., Smith et al. 1996). In addition, among its RGB stars, large ${ }^{3} \mathrm{O}$ differences are present (anticorrelated with $\mathrm{N}$ ), as well as significant star-to-star scatter in $\mathrm{Na}$ and $\mathrm{Al}$ ( $\mathrm{Na}$ anticorrelated with $\mathrm{Mg}$ and correlated with O; see Kraft et al. 1997; Cavallo \& Nagar 2000). Such observations are suggestive of the mixing of proton-exposed material to the surfaces of some cluster members, and there is evidence of shifts toward lower $\mathrm{O}$ and higher $\mathrm{Na}$ and $\mathrm{Al}$ abundances among the most luminous RGB stars $\left(M_{V}<-1.7\right.$; Pilachowski et al. 1996; Kraft et al. 1997; Cavallo \& Nagar 2000). Yet, at the same time a large dispersion in these elements is present in the data of Kraft et al. to within

\footnotetext{
${ }^{3}$ As commented by Kraft et al. (1997), the M13 RGB stars exhibit some of the largest inhomogeneities known.
}

at least $0.25 \mathrm{mag}$ of the luminosity function (LF) bump at $V \approx 14.75$ (Paltrinieri et al. 1998). A large scatter in $\mathrm{C}$ and $\mathrm{N}$ abundances was also followed to a similar luminosity by Suntzeff (1981). Grundahl, VandenBerg, \& Andersen (1998) found scatter in Strömgren photometry among the RGB and SGB stars in M13 that they attributed to a spread in CNO abundances probably extending at least to the MSTO.

We report here on recent observations and derived $\mathrm{C}$ abundances of a sample of SGB/MS stars in the globular cluster M13 that appear to support the hypothesis of a substantial intrinsic spread in the distribution of light elements within the cluster, which is further modified during RGB ascent.

\section{OBSERVATIONS}

The construction of the photometric catalog used here for M13 is described in Stetson, Hesser, \& Smecker-Hane (1998) and Stetson (2000) and is similar to that built for M5 and described in detail in our earlier work (Cohen et al. 2002). Slit masks were designed focusing on the subgiants in M13 and on the region just below the turnoff. We note that the base of the RGB in M13 is at about $V=17.7, I=16.9$, while the MSTO is at about $V=18.2, I=17.8$.

These slit masks were used on the blue side (McCarthy et al. 1998) of the Low-Resolution Imaging Spectrometer (LRIS; Oke et al. 1995) at the Keck Telescope in 2001 May and 2002 May. As the weather was suboptimal, four $1000 \mathrm{~s}$ exposures were obtained with the slit mask intended for the fainter stars. The $600 \mathrm{~g} \mathrm{~mm}^{-1}$ grism was used with a 0.7 wide slit, yielding a dispersion of $1.0 \AA$ pixel $^{-1}$ and a spectral resolution of $4 \AA$. The spectral coverage was from 3500 to $5500 \AA$ for essentially all the stars. There are 81 stars with spectra good enough for the present purposes, namely, more than $450 \mathrm{ADU}_{\text {pixel }}^{-1}$ (with $\left.2 e^{-} \mathrm{ADU}^{-1}\right)$ in the continuum at $4360 \AA$.

Nonmembers are very easily recognized owing to the low metallicity of M13 and its large negative radial velocity. Since the stars were selected to lie on the cluster isochrone and M13 is at high galactic latitude, one would expect very few nonmembers. All of the stars in the sample presented here are members of M13. Full details of the sample of stars and the observations will be given in a forthcoming paper.

\section{ANALYSIS}

Our analysis essentially parallels that of Briley \& Cohen (2001, hereafter BC01), and the reader is referred to that work for details. The strengths of the $4350 \AA \mathrm{CH}(G)$ bands of our program stars were measured via the $I(\mathrm{CH})$ index of Cohen (1999a, 1999b) - a double-sided index that compares the flux removed by the $G$ band to the adjacent continuum. The resulting indices (corrected for the radial velocity of M13) are plotted as a function of $I$ magnitude in Figure 1, where a significant spread among both the SGB and MS stars can be seen. As is discussed in BC01, the decrease in the spread in $I(\mathrm{CH})$ near $I \approx 18$ is the result of the high temperatures and low metallicities of the M13 MSTO stars (i.e., there simply is little $\mathrm{CH}$ formation in such stars).

In order to relate the observed indices to the underlying [C/Fe], we employ a series of synthetic spectra based on MARCS (Gustafsson et al. 1975) model atmospheres. We assume that the iron abundance is constant within M13 as suggested by the highdispersion spectroscopic studies of low-luminosity globular cluster samples by Gratton et al. (2001) and by Ramírez \& Cohen (2002a, 2002b). Our MSTO models are those used in BC01 to which we have added an additional three luminous SGB models 


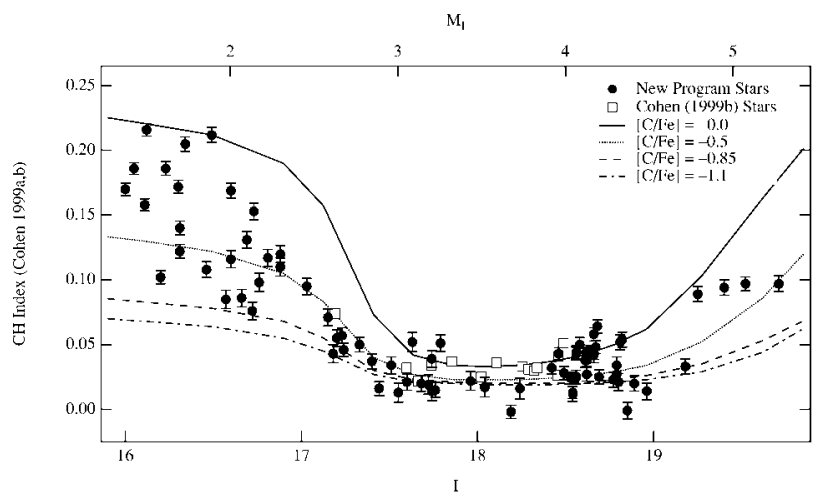

FIG. 1.-Observed and synthetic $I(\mathrm{CH})$ indices for stars in M13 are plotted as a function of luminosity. Also plotted are the indices from Cohen (1999a); $1 \sigma$ error bars based on Poisson statistics are included. A large spread in $I(\mathrm{CH})$ is present for both the SGB and pre-MSTO stars, as well as among the stars at the base of the turnoff. Using the model isoabundance lines as a guide, the scatter in $I(\mathrm{CH})$ appears consistent with a constant range in $[\mathrm{C} / \mathrm{Fe}]$ over the entire luminosity range.

(at $T_{\text {eff }} / \log g=5168 / 2.96,5224 / 3.15,5281 / 3.33$ ) and five fainter MS points (at 6118/4.47, 6034/4.53, 5833/4.59, 5601/4.66, $5351 / 4.70)$ taken from the $16 \mathrm{Gyr}[\mathrm{Fe} / \mathrm{H}]=-1.48 \mathrm{O}$-enhanced isochrone grid of Bergbusch \& Vandenberg (1992). As in BC01, we adopt a geometric distance of $7.2 \mathrm{kpc}$ [plus an additional 0.11 magnitudes in $\left.(m-M)_{V}\right]$ and a reddening of $E(B-V)=$ 0.02 mag following Harris (1996).

From each model and a given set of C/N/O abundances, synthetic spectra were computed using the SSG program (Bell, Paltoglou, \& Tripicco 1994 and references therein) and the line list of Tripicco \& Bell (1995; see BC01 for further details). The result was then convolved with a Gaussian to match the resolution of the observed spectra and $I(\mathrm{CH})$ indices measured. Four compositions are illustrated here $([\mathrm{C} / \mathrm{Fe}] /[\mathrm{N} / \mathrm{Fe}] /[\mathrm{O} / \mathrm{Fe}])$ : $-0.85 /+0.7 /+0.4$ and $-1.1 /+1.2 /-0.5$, which (as in BC01) roughly match the observed compositions of M13's CN-weak and $\mathrm{CN}$-strong bright giants, respectively, $0.0 /+0.4 /+0.4$, an arbitrary "premixing" composition, and $-0.5 /+1.4 / 0.0$, a redistribution of the "premixing" composition holding the sum of $\mathrm{C}+\mathrm{N}+\mathrm{O}$ constant. The curves plotted in Figure 1 are $I(\mathrm{CH})$ indices calculated from these synthetic spectra, where it can be seen that the small scatter in $I(\mathrm{CH})$ among the MSTO stars is due to the intrinsic weakness of the $G$ band rather than an absence of inhomogeneities (as pointed out by BC01).

Note that among such warm relatively metal poor stars, the resulting indices are remarkably insensitive to all but our choice of $[\mathrm{C} / \mathrm{Fe}]$. This is the result of the low metallicity; $\mathrm{CN}$ and $\mathrm{CO}$ play a less important role in the molecular equilibrium for carbon. For example, using the 5281/3.33 model $\left(M_{I}=+1.89\right)$ with $-0.5 /+1.4 / 0.0$ as a baseline, increasing $[\mathrm{O} / \mathrm{Fe}]$ by $0.4 \mathrm{dex}$ decreases $I(\mathrm{CH})$ by 0.006 , decreasing $[\mathrm{N} / \mathrm{Fe}]$ by 1.4 dex increases $\mathrm{I}(\mathrm{CH})$ by 0.006 , and increasing ${ }^{12} \mathrm{C} /{ }^{13} \mathrm{C}$ to 90 has essentially no effect. The greatest sensitivity appears to be to microturbulence $\left(v_{t}\right)$, as increasing $v_{t}$ from 1.5 to $2.5 \mathrm{~km} \mathrm{~s}^{-1}$ increases $I(\mathrm{CH})$ by 0.010 (an $8 \%$ increase), which is negligible compared to the changes resulting from different values of $[\mathrm{C} / \mathrm{Fe}]$.

We have applied the method of Briley et al. (1990) to convert the observed $I(\mathrm{CH})$ indices to corresponding $\mathrm{C}$ abundances: the model isoabundance curves were interpolated to the $M_{I}$ of each program star, and the observed $I(\mathrm{CH})$ index converted into $[\mathrm{C} / \mathrm{Fe}]$ based on the synthetic indices at that $M_{I}$. As such, we are assuming that a given star's $M_{I}$ maps to the $T_{\text {eff }}$, $\log g$, and mass specified by the Bergbusch \& Vandenberg

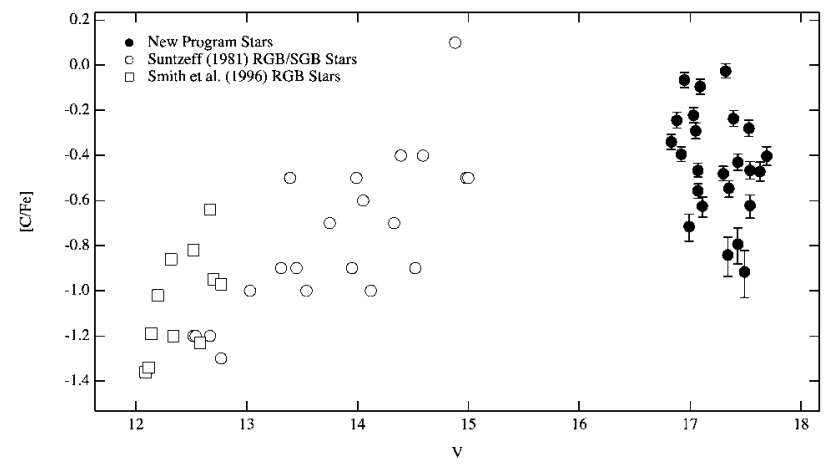

FIG. 2.-Carbon abundances derived from the present SGB stars in M13 are plotted with the results of Suntzeff (1981) and Smith et al. (1996). Although a large and consistent scatter in $[\mathrm{C} / \mathrm{Fe}]$ apparently exists from the SGB to the tip of the $\mathrm{RGB}$, the decline in $[\mathrm{C} / \mathrm{Fe}]$ with luminosity near the $\mathrm{RGB}$ tip is striking.

(1992) isochrone based on our calculation of model luminosity/ color and choice of distance modulus and reddening. Returning to the 5281/3.33 model (the faintest model used in deriving $\mathrm{SGB}[\mathrm{C} / \mathrm{Fe}]$ ), we note that an underestimation in $T_{\text {eff }}$ of $100 \mathrm{~K}$ would correspond to an error of +0.03 in $V-I$ and a derived $[\mathrm{C} / \mathrm{Fe}]$ some 0.12 dex too large at $[\mathrm{C} / \mathrm{Fe}]=-0.5$. Likewise, an underestimation of $\log g$ by 0.1 dex would be equivalent to a shift of -0.25 in $M_{I}$ and $-0.08 \mathrm{dex}$ in $[\mathrm{C} / \mathrm{Fe}]$. Essentially identical changes result when considering our most luminous SGB model (5168/2.96). Thus, if one takes $100 \mathrm{~K}$ and $0.1 \mathrm{dex}$ as reasonable errors in $T_{\text {eff }}$ and $\log g$, we estimate our uncertainties in $[\mathrm{C} / \mathrm{Fe}]$ to be roughly $0.1 \mathrm{dex}$.

\section{RESULTS}

The resulting $[\mathrm{C} / \mathrm{Fe}]$ values for the subgiants only are plotted in Figure 2, as well as the $[\mathrm{C} / \mathrm{Fe}]$ values derived by Suntzeff (1981) and Smith et al. (1996) for more luminous cluster members. Immediately obvious from Figure 2 is a marked decline in [C/Fe] with increasing luminosity among the M13 giants-the mean $[\mathrm{C} / \mathrm{Fe}]$ of Sunzteff's "RGB" sample is $-0.9 \pm 0.3$ dex, while for the present SGB stars the value is $-0.4 \pm-0.2$ dex. This pattern in $[\mathrm{C} / \mathrm{Fe}]$ was noted by Suntzeff, but the depletions appear to set in at a luminosity below the limit of his sample. Such a result can most easily be understood in the context of the deep-mixing framework where C-depleted material from the $\mathrm{CN}$-burning region above the $\mathrm{H}$-burning shell is progressively circulated into the stellar envelope as discussed above. We note however that changes in the distribution of $\mathrm{O}, \mathrm{Na}$, and $\mathrm{Al}$ in M13 are suggested by Pilachowski et al. (1996), Kraft et al. (1997), and Cavallo \& Nagar (2000) to occur only among its most luminous giants, i.e., $V<13$, which is also the point at which the $\mathrm{C}$ abundances drop precipitously in Figure 2. It would thus appear that during the bulk of RGB ascent, mixing only into $\mathrm{CN}$ cycle regions is taking place in M13.

At the same time, a large scatter in $[\mathrm{C} / \mathrm{Fe}]$ (roughly $0.8 \mathrm{dex}$ ), comparable to that found among the more luminous stars, is present in the LRIS SGB sample in Figure 2. Figure 1 further suggests that a large dispersion in $\mathrm{C}$ abundance is also present at luminosities below the MSTO. (We have no information on $\mathrm{N}$ abundances at present.) These inhomogeneities are in place well below the LF bump and the operation of proposed deepmixing mechanisms. Thus, it appears that the action of an external process has shaped much of the star-to-star scatter present in M13, i.e., the large range of $\mathrm{C}$, and likely other light abundances found among the giants (at least fainter than 
$V=13$ ) is to a great extent not the result of varying mixing efficiencies within the stars themselves.

If we assume the initial $[\mathrm{C} / \mathrm{Fe}]$ values to be roughly solar in the proto-M13 gas cloud, the $\mathrm{C}$-poor stars in our sample might be explained by the incorporation of C-poor, N-rich AGB ejecta. However, the large spread in $\mathrm{C}$ abundances seen here requires far more than the "pollution" of the stellar surfaces by simple accretion of AGB ejecta - such surface contaminations would be quickly erased by the deepening convective envelope following the MS phase. This is clearly not observed, and a substantial fraction of the stars' total mass must therefore be homogeneous in these elements. This in turn requires the accretion of not just of a sprinkling but rather of a significant amount of material: if we consider a typical C-poor M13 star $([\mathrm{C} / \mathrm{Fe}]=-0.6)$, some $70 \%$ of the star's mass must be captured ejecta (assuming the accreted matter to be completely free of $\mathrm{C}$ ). While this initially seems implausible, we note the recent calculations of Thoul et al. (2002) that demonstrate that large accumulations may be possible, particularly in clusters with small core radii, e.g., 47 Tuc, where they estimate as much as $80 \%$ of the mass of a $1 M_{\odot}$ star could be accreted material. Unfortunately, the core radius of M13 is considerably larger, and similar calculations yield an expected accretion of only $8 \%$. Whether this is a problem that can be overcome with more detailed accretion models (e.g., Thoul et al. included several assumptions, such as chaotic orbits with stars spending $20 \%$ of their time in the cluster core, which was also the spatial extent of the central gas reservoir), a reflection of changes in the structure/dynamics of M13 since its formation, or can be better explained by an alternative mechanism remains to be seen.

The work presented here implies we are seeing the effects of a significant external "contamination" of unexplained origin upon which a poorly understood mixing mechanism acts during the later stages of RGB ascent. However, there remain a number of issues to address: The critical region just fainter than the M13 LF bump at $V \approx 14.75$ has yet to be explored, leaving the exact luminosity of $\mathrm{C}$ depletion onset uncertain. Also, in comparing the present results with those of Suntzeff (1981) and Smith et al. (1996), we are comparing abundances based on different instruments, indices, and analysis tools-note the apparent 0.3 dex offset between the $\mathrm{C}$ abundances of Suntzeff and Smith in Figure 2. Furthermore, we urgently need a larger sample of stars below the MSTO in M13. To address these issues, we will be returning to M13 in the near future.

The entire Keck/LRIS user communities owes a huge debt to Jerry Nelson, Gerry Smith, Bev Oke, and many other people who have worked to make the Keck Telescope and LRIS a reality and to operate and maintain the Keck Observatory. We are grateful to the W. M. Keck Foundation for the vision to fund the construction of the W. M. Keck Observatory. We wish to express our thanks to Roger Bell, whose SSG code was instrumental in this project. Partial support was provided by the National Science Foundation under grant AST 00-98489 to M. M. B. and grants AST 98-19614 and AST 02-05951 to J. G. C. and by the F. John Barlow professorship (M. M. B.).

\section{REFERENCES}

Aikawa, M., Fujimoto, M. Y., \& Kato, K. 2001, ApJ, 560, 937

Bell, R. A., Paltoglou, G., \& Tripicco, M. J. 1994, MNRAS, 268, 771

Bellman, S., Briley, M. M., Smith, G. H., \& Claver, C. F. 2001, PASP, 113, 326

Bergbusch, P. A., \& Vandenberg, D. A. 1992, ApJS, 81, 163

Briley, M. M., Bell, R. A., Hoban, S., \& Dickens, R. J. 1990, ApJ, 359, 307

Briley, M. M., \& Cohen, J. G. 2001, AJ, 122, 242 (BC01)

Briley, M. M., Smith, V. V., Suntzeff, N. B., Lambert, D. L., Bell, R. A., \& Hesser, J. E. 1996, Nature, 383, 604

Cannon, R. D., Croke, B. F. W., Bell, R. A., Hesser, J. E., \& Stathakis, R. A. 1998, MNRAS, 298, 601

Cavallo, R. M., \& Nagar, N. M. 2000, AJ, 120, 1364

Charbonnel, C. 1995, ApJ, 453, L41

Charbonnel, C., \& do Nascimento, J. D. 1998, A\&A, 336, 915

Cohen, J. G. 1999a, AJ, 117, 2428 1999b, AJ, 117, 2434

Cohen, J. G., Briley, M. M., \& Stetson, P. B. 2002, AJ, 123, 2525

Cottrell, P. L., \& Da Costa, G. S. 1981, ApJ, 245, L79

Da Costa, G. S. 1998, in IAU Symp. 189, Fundamental Stellar Properties: The Interaction between Observation and Theory, ed. T. R. Bedding, A. J. Booth, \& J. Davis (Dordrecht: Kluwer), 193

Denissenkov, P. A., \& Tout, C. A. 2000, MNRAS, 316, 395

Gratton, R. G., Sneden, C., Carretta, E., \& Bragaglia, A. 2000, A\&A, 354, 169

Gratton, R. G., et al. 2001, A\&A, 369, 87

Grundahl, F., VandenBerg, D. A., \& Andersen, M. L. 1998, ApJ, 500, L179

Gustafsson, B., Bell, R. A., Eriksson, K., \& Nordlund, A. 1975, A\&A, 42, 407

Harris, W. E. 1996, AJ, 112, 1487
Hesser, J. E. 1978, ApJ, 223, L117

Keller, L. D., Pilachowski, C. A., \& Sneden, C. 2001, AJ, 122, 2554

Kraft, R. P. 1994, PASP, 106, 553

Kraft, R. P., Sneden, C., Langer, G. E., \& Prosser, C. F. 1992, AJ, 104, 645

Kraft, R. P., Sneden, C., Smith, G. H., Shetrone, M. D., Langer, G. E., \& Pilachowski, C. A. 1997, AJ, 113, 279

McCarthy, J. K., et al. 1998, Proc. SPIE, 3355, 81

Oke, J. B., et al. 1995, PASP, 107, 375

Paltrinieri, B., Ferraro, F. R., Carretta, E., \& Fusi Pecci, F. 1998, MNRAS, 293, 434

Pilachowski, C. A., Sneden, C., Kraft, R. P., \& Langer, G. E. 1996, AJ, 112 , 545

Ramírez, S. V., \& Cohen, J. G. 2002a, AJ, 123, 3277 2002b, AJ, submitted

Salaris, M., Cassisi, S., \& Weiss, A. 2002, PASP, 114, 375

Smith, G. H., Shetrone, M. D., Bell, R. A., Churchill, C. W., \& Briley, M. M. 1996, AJ, 112, 1511

Stetson, P. B. 2000, PASP, 112, 925

Stetson, P. B., Hesser, J. E., \& Smecker-Hane, T. 1998, PASP, 110, 533

Suntzeff, N. B. 1981, ApJS, 47, 1

Suntzeff, N. B., \& Smith, V. V. 1991, ApJ, 381, 160

Sweigart, A. V., \& Mengel, J. G. 1979, ApJ, 229, 624

Thoul, A., Jorissen, A., Goriely, S., Jehin, E., Magain, P., Noels, A., \& Parmentier, G. 2002, A\&A, 383, 491

Trefzger, C. F., Carbon, D. F., Langer, G. E., Suntzeff, N. B., \& Kraft, R. P. 1983, ApJ, 266, 144

Tripicco, M. J., \& Bell, R. A. 1995, AJ, 110, 3035

Ventura, P., D’Antona, F., Mazzitelli, I., \& Gratton, R. 2001, ApJ, 550, L65 